\title{
GOURAVA AND HYPER-GOURAVA INDICES OF SOME CACTUS CHAINS
}

\author{
B. BASAVANAGOUD ${ }^{1}$ AND SHRUTI POLICEPATIL ${ }^{2}$ \\ ${ }^{1,2}$ Department of Mathematics, Karnatak University \\ Dharwad - 580003 Karnataka, India \\ ${ }^{1}$ b.basavanagoud@gmail.com \\ ${ }^{2}$ shrutipatil300@gmail.com
}

\begin{abstract}
The physico-chemical characteristics of molecules are theoretically explored using the theory of graphs and mathematical chemistry. A graph's topological index is a numerical value derived from the graph mathematically. The Gourava and hyper-Gourava indices of various cactus chains are determined in this study.

Key words and Phrases: Gourava indices, hyper-Gourava indices, cactus chains.
\end{abstract}

\section{INTRODUCTION}

A molecular graph, also known as a chemical graph, is a graph in which the atoms are represented by the vertices, while the bonds are represented by the edges. Topological indices are numeric quantities obtained from a molecular graph that correlate the molecular graph's physico-chemical characteristics and have been shown to be beneficial in isomer discrimination, QSAR and QSPR analysis.

Only simple, finite, connected graphs with $V(G)$ as vertex set and $E(G)$ as edge set are considered throughout this study. The degree $d_{G}(a)$ of a vertex $a$ is the number of vertices adjacent to $a$.

A cactus graph is a connected graph in which no edge lies in more than one cycle. Every cactus graph cycle is chordless, and every cactus graph block is either an edge or a cycle. A cactus graph is said to be triangular if all of its blocks are triangular. A triangular cactus graph is described as a chain triangular cactus if all of its triangles have at most two cut-vertices and each cut-vertice is shared by precisely two triangles. A square cactus graph is a type of cactus graph and all of its blocks are square. A square cactus graph is said to be a chain square cactus if all of its squares have at most two cut-vertices and each cut-vertice is shared by precisely two squares. It's worth noting that the internal squares' connections

2020 Mathematics Subject Classification: 05C07, $05 \mathrm{C} 09$.

Received: 25-02-2021, accepted: 28-08-2021. 
to their neighbours may vary. A chain square cactus is called ortho-chain square cactus if the cut-vertices are nearby. A para-chain square cactus is one in which the cut-vertices are not contiguous in a chain square cactus. The Gourava and hyperGourava indices of various generic ortho and para cactus chains are studied in this paper, and particular situations such as the triangular chain cactus $T_{n}$, ortho-chain square cactus $O_{n}$, and para-chain square cactus $Q_{n}$ are considered. Latest investigations on several cactus chains can be found in $[1,3,13,14]$ and references cited therein. For undefined terms and notations refer to [5].

The first and second Gourava indices of a molecular graph were introduced by Kulli [6] and are defined as:

$$
\begin{aligned}
& G O_{1}(G)=\sum_{a b \in E(G)}\left[\left(d_{G}(a)+d_{G}(b)\right)+d_{G}(a) d_{G}(b)\right], \\
& G O_{2}(G)=\sum_{a b \in E(G)}\left[\left(d_{G}(a)+d_{G}(b)\right)\left(d_{G}(a) d_{G}(b)\right)\right] .
\end{aligned}
$$

Kulli proposed the first and second hyper-Gourava indices of a molecular graph $G$ in [7], and they are defined as

$$
\begin{aligned}
H G O_{1}(G) & =\sum_{a b \in E(G)}\left[d_{G}(a)+d_{G}(b)+d_{G}(a) d_{G}(b)\right]^{2}, \\
H G O_{2}(G) & =\sum_{a b \in E(G)}\left[\left(d_{G}(a)+d_{G}(b)\right)\left(d_{G}(a) d_{G}(b)\right)\right]^{2} .
\end{aligned}
$$

Several topological indices were investigated. For further information, see $[2,4,8$, $9,10,11,12]$.

\section{MAIN RESULTS}

We look at two types of cactus chains in this section: the para cacti chain and the ortho cacti chain of cycles. We start with a para cacti chain of length $n$ cycles $C_{m}$, where each block is a cycle $C_{m}$. Let $C_{m}^{n}$ be the symbol for it. We compute an exact expression of $G O_{1}, G O_{2}, H G O_{1}$ and $H G O_{2}$ of $C_{m}^{n}$ in the following theorem.

TABLE 1. Partitioning at the edge of $C_{m}^{n}$.

\begin{tabular}{|c|c|c|}
\hline$d_{C_{m}^{n}}(a), d_{C_{m}^{n}}(b): a b \in E\left(C_{m}^{n}\right)$ & $(2,2)$ & $(2,4)$ \\
\hline Edge count & $m n-4 n+4$ & $4(n-1)$ \\
\hline
\end{tabular}

Theorem 2.1. For a para cacti chain of cycles $C_{m}^{n}(m \geq 4, n \geq 2)$,

1. $G O_{1}\left(C_{m}^{n}\right)=8[m n+3(n-1)]$. 
2. $G O_{2}\left(C_{m}^{n}\right)=16[m n+8(n-1)]$.

3. $H G O_{1}\left(C_{m}^{n}\right)=16[4 m n+33(n-1)]$.

4. $\mathrm{HGO}_{2}\left(C_{m}^{n}\right)=256[m n+32(n-1)]$.

Proof. 1. By utilizing the definition of $G O_{1}$ and entries in Table 1, we have

$$
\begin{aligned}
G O_{1}\left(C_{m}^{n}\right) & =\sum_{a b \in E\left(C_{m}^{n}\right)}\left[\left(d_{C_{m}^{n}}(a)+d_{C_{m}^{n}}(b)\right)+\left(d_{C_{m}^{n}}(a) d_{C_{m}^{n}}(b)\right)\right] \\
& =(m n-4 n+4)(4+4)+4(n-1)(2+4+8) \\
& =8[m n+3(n-1)] .
\end{aligned}
$$

2. By making use the definition of $G_{2}$ and values in Table 1, we have

$$
\begin{aligned}
G O_{2}\left(C_{m}^{n}\right) & =\sum_{a b \in E\left(C_{m}^{n}\right)}\left[\left(d_{C_{m}^{n}}(a)+d_{C_{m}^{n}}(b)\right)\left(d_{C_{m}^{n}}(a) d_{C_{m}^{n}}(b)\right)\right] \\
& =(m n-4 n+4)(4 \times 4)+4(n-1)(6 \times 8) \\
& =16[m n+8(n-1)] .
\end{aligned}
$$

3. By the usage of the definition of $H G O_{1}$ and facts in table 1 , we have

$$
\begin{aligned}
H G O_{1}\left(C_{m}^{n}\right) & =\sum_{a b \in E\left(C_{m}^{n}\right)}\left[\left(d_{C_{m}^{n}}(a)+d_{C_{m}^{n}}(b)\right)+\left(d_{C_{m}^{n}}(a) d_{C_{m}^{n}}(b)\right)\right]^{2} \\
& =(m n-4 n+4)(4+4)^{2}+4(n-1)(6+8)^{2} \\
& =16[4 m n+33(n-1)] .
\end{aligned}
$$

4. By using the concept of $H G O_{2}$ as well as the data in Table 1 , we have

$$
\begin{aligned}
H G O_{2}\left(C_{m}^{n}\right) & =\sum_{a b \in E\left(C_{m}^{n}\right)}\left[\left(d_{C_{m}^{n}}(a)+d_{C_{m}^{n}}(b)\right)\left(d_{C_{m}^{n}}(a) d_{C_{m}^{n}}(b)\right)\right]^{2} \\
& =(m n-4 n+4)(4 \times 4)^{2}+4(n-1)(6 \times 8)^{2} \\
& =256[m n+32(n-1)] .
\end{aligned}
$$

The graph $Q_{n}$ is pictured in Figure 1.

Corollary 2.2. For a para-chain square cactus graph $Q_{n}(n \geq 2)$,

1. $G O_{1}\left(Q_{n}\right)=8(7 n-3)$. 


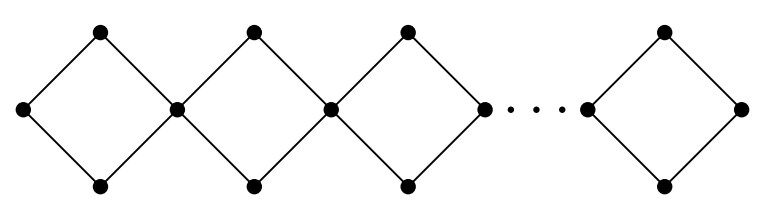

Figure 1. The graph $Q_{n}$.

2. $G O_{2}\left(Q_{n}\right)=3 n^{3}+9 n^{2}+60 n$.

3. $H G O_{1}\left(Q_{n}\right)=16(49 n-33)$.

4. $\mathrm{HGO}_{2}\left(Q_{n}\right)=1024(9 n-8)$.

Proof. Replace $m=4$ in Theorem 2.1 to complete the proof.

The graph $L_{n}$ is indicated in Figure 2.

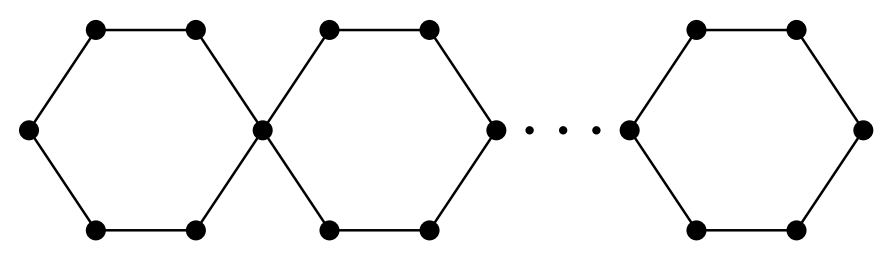

FiguRE 2. The graph $L_{n}$.

Corollary 2.3. For a para-chain hexagonal cactus graph $L_{n}(n \geq 3)$,

1. $G O_{1}\left(L_{n}\right)=24(3 n-1)$.

2. $\mathrm{GO}_{2}\left(L_{n}\right)=32(7 n-4)$.

3. $H G O_{1}\left(L_{n}\right)=16(57 n-33)$.

4. $\mathrm{HGO}_{2}\left(L_{n}\right)=512(19 n-16)$.

ProOF. We get the required outcome if we set $m=6$ in the Theorem 2.1.

The ortho-chain cacti of cycles with neighbouring cut-vertices is now considered. Let $C O_{m}^{n}$ be an ortho-chain cactus graph, where $m$ is the cycle length and $n$ is the chain length. $\left|V\left(C O_{m}^{n}\right)\right|=m n-n+1$ and $\left|E\left(C O_{m}^{n}\right)\right|=m n$ are self-evident. $G O_{1}, G O_{2}, H G O_{1}$ and $\mathrm{HGO}_{2}$ of $C O_{m}^{n}$ are obtained by utilizing the following theorem.

TABle 2. Partitioning at the edge of $C O_{m}^{n}$.

\begin{tabular}{|c|c|c|c|}
\hline$d_{C O_{m}^{n}}(a), d_{C O_{m}^{n}}(b): a b \in E\left(C O_{m}^{n}\right)$ & $(2,2)$ & $(2,4)$ & $(4,4)$ \\
\hline Edge count & $m n-3 m+2$ & $2 n$ & $n-1$ \\
\hline
\end{tabular}

Theorem 2.4. For a ortho cacti chain of cycles $C O_{m}^{n}(m \geq 3, n \geq 2)$,

1. $G O_{1}\left(C O_{m}^{n}\right)=8 m n-24 m+52 n-8$. 
2. $\mathrm{GO}_{2}\left(C \mathrm{O}_{m}^{n}\right)=16 m n-48 m+224 n-96$.

3. $H G O_{1}\left(C O_{m}^{n}\right)=64 m n-192 m+968 n-448$.

4. $\mathrm{HGO}_{2}\left(\mathrm{CO}_{m}^{n}\right)=256 m n-768 m+20992 n-15872$.

Proof. 1. By using the concept of $G O_{1}$ as well as the data in Table 2, we have

$$
\begin{aligned}
G O_{1}\left(C O_{m}^{n}\right) & =\sum_{a b \in E\left(C O_{m}^{n}\right)}\left[\left(d_{C O_{m}^{n}}(a)+d_{C O_{m}^{n}}(b)\right)+\left(d_{C O_{m}^{n}}(a) d_{C O_{m}^{n}}(b)\right)\right] \\
& =(m n-3 m+2)(4+4)+2 n(6+8)+(n-1)(8+16) \\
& =8 m n-24 m+52 n-8 .
\end{aligned}
$$

2. By making use the definition of $\mathrm{GO}_{2}$ and values in Table 2, we have

$$
\begin{aligned}
G_{2}\left(C O_{m}^{n}\right) & =\sum_{a b \in E\left(C O_{m}^{n}\right)}\left[\left(d_{C O_{m}^{n}}(a)+d_{C O_{m}^{n}}(b)\right)+\left(d_{C O_{m}^{n}}(a) d_{C O_{m}^{n}}(b)\right)\right] \\
& =(m n-3 m+2)(4 \times 4)+2 n(6 \times 8)+(n-1)(8 \times 16) \\
& =16 m n-48 m+224 n-96 .
\end{aligned}
$$

3. By utilizing the description of $H G O_{1}$ and entries in Table 2, we have

$$
\begin{aligned}
H G O_{1}\left(C O_{m}^{n}\right) & =\sum_{a b \in E\left(C O_{m}^{n}\right)}\left[\left(d_{C O_{m}^{n}}(a)+d_{C O_{m}^{n}}(b)\right)+\left(d_{C O_{m}^{n}}(a) d_{C O_{m}^{n}}(b)\right)\right]^{2} \\
& =(m n-3 m+2)(4+4)^{2}+2 n(6+8)^{2}+(n-1)(8+16)^{2} \\
& =64 m n-192 m+968 n-448 .
\end{aligned}
$$

4. By the usage of the definition of $\mathrm{HGO}_{2}$ and facts in table 2, we have

$$
\begin{aligned}
H_{G O_{2}}\left(C O_{m}^{n}\right) & =\sum_{a b \in E\left(C O_{m}^{n}\right)}\left[\left(d_{C O_{m}^{n}}(a)+d_{C O_{m}^{n}}(b)\right)+\left(d_{C O_{m}^{n}}(a) d_{C O_{m}^{n}}(b)\right)\right]^{2} \\
& =(m n-3 m+2)(4 \times 4)^{2}+2 n(6 \times 8)^{2}+(n-1)(8 \times 16)^{2} \\
& =256 m n-768 m+20992 n-15872 .
\end{aligned}
$$

Then, as illustrated in Figure 3, we consider a chain triangular cactus, designated by $T_{n}$, where $n$ is the length of the $T_{n}$. For $m=3, T_{n}$ is a special case of $C O_{m}^{n}$. 


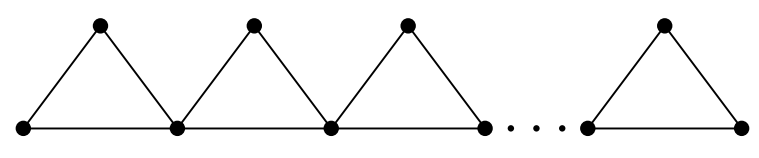

Figure 3. The graph $T_{n}$.

Corollary 2.5. For a chain triangular cactus $T_{n}(n \geq 2)$,

1. $G O_{1}\left(T_{n}\right)=76 n-80$.

2. $G O_{2}\left(T_{n}\right)=272 n-240$.

3. $H G O_{1}\left(T_{n}\right)=1160 n-1024$.

4. $H_{G O} O_{2}\left(T_{n}\right)=21760 n-18176$.

Proof. Replace $m=3$ in Theorem 2.4 to complete the proof.

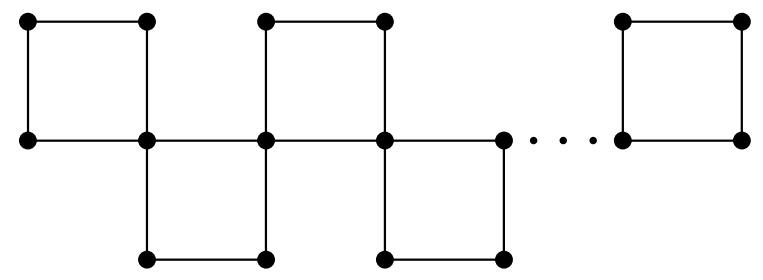

Figure 4. The graph $O_{n}$.

Corollary 2.6. For the ortho-chain square cactus $O_{n}(n \geq 2)$,

1. $G O_{1}\left(O_{n}\right)=84 n-104$.

2. $G O_{2}\left(O_{n}\right)=288(n-1)$.

3. $H G O_{1}\left(O_{n}\right)=1224 n-1216$.

4. $\mathrm{HGO}_{2}\left(\mathrm{O}_{n}\right)=22016 n-18944$.

Proof. We get the required outcome if we set $m=4$ in the Theorem 2.4.

By identifying every node of $K_{m}$ with a node of one $K_{y}$, the graph $Q(m, y)$ is formed from $K_{m}$ and $m$ copies of $K_{y} . G O_{1}, G O_{2}, H G O_{1}$ and $H G O_{2}$ of $Q(m, y)$ are computed in the following theorem. Figure 5 depicts the graph $Q(m, y)$.

TABLE 3. Partitioning at the edge of $Q(m, y)$.

\begin{tabular}{|c|c|}
\hline$d_{Q(m, y)}(a), d_{Q(m, y)}(b): a b \in E(Q(m, y))$ & Edge count \\
\hline$(y-1, y-1)$ & $\frac{m(y-1)(y-2)}{2}$ \\
\hline$(y-1, m+y-2)$ & $m(y-1)$ \\
\hline$(m+y-2, m+y-2)$ & $\frac{m(y-1)}{2}$ \\
\hline
\end{tabular}

Theorem 2.7. For a ortho-chain $Q(m, y)(m, y \geq 2)$, 


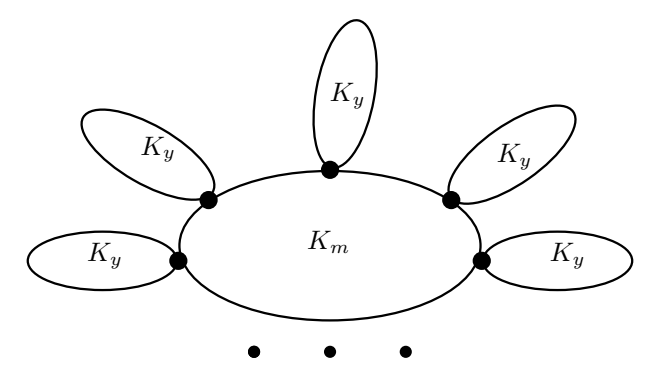

Figure 5. The graph $Q(m, y)$.

1. $G O_{1}(Q(m, y))=\frac{m(y-1)(y-2)\left(y^{2}-1\right)}{2}+m(y-1)\left(y^{2}+y m-y-1\right)$

$+\frac{m(y-1)(m+y-2)(m+y)}{2}$.

2. $G O_{2}(Q(m, n))=m(y-1)^{4}(y-2)+m(y-1)\left[\left(2 y^{2}-9 y+13\right)+m(5-m)\right.$

$+m y(3 y+m-8)-6]+m(y-1)\left[(m+y-2)^{3}\right]$.

3. $H G O_{1}(Q(m, y))=\frac{m(y-1)(y-2)\left(y^{2}-1\right)^{2}}{2}+m(y-1)\left(y^{2}+y m-y-1\right)^{2}$

$+\frac{m(y-1)(m+y-2)(m+y)^{2}}{2}$.

4. $H G O_{2}(Q(m, y))=2 m(y-1)\left[(y-1)^{6}(y-2)+(m+y-2)^{6}\right]$ $+m(n-1)[(m+2 y-3)(y-1)(m+y-2)]^{2}$.

Proof. 1. By using the concept of $G O_{1}$ as well as the data in Table 3, we have

$$
\begin{aligned}
G O_{1}(Q(m, y))= & \sum_{a b \in E(Q(m, y))}\left[\left(d_{Q(m, y)}(a)+d_{Q(m, y)}(b)\right)+\left(d_{Q(m, y)}(a) d_{Q(m, y)}(b)\right)\right] \\
= & \frac{m(y-1)(y-2)\left(y^{2}-1\right)}{2}+m(y-1)\left(y^{2}+y m-y-1\right) \\
& +\frac{m(y-1)(m+y-2)(m+y)}{2} .
\end{aligned}
$$

2. By utilizing the description of $\mathrm{GO}_{2}$ and entries in Table 3, we have

$$
\begin{aligned}
G O_{2}(Q(m, n))= & \sum_{a b \in E(Q(m, n))}\left[\left(d_{Q(m, y)}(a)+d_{Q(m, y)}(b)\right)\left(d_{Q(m, y)}(a) d_{Q(m, y)}(b)\right)\right] \\
= & m(y-1)^{4}(y-2)+m(n-1)\left[\left(2 y^{2}-9 y+13\right)+m(5-m)\right. \\
& +m y(3 y+m-8)-6]+m(y-1)\left[(m+y-2)^{3}\right] .
\end{aligned}
$$

3. By the usage of the definition of $H G O_{1}$ and facts in Table 3, we have 


$$
\begin{aligned}
H G O_{1}(Q(m, y))= & \sum_{a b \in E(Q(m, y))}\left[\left(d_{Q(m, y)}(a)+d_{Q(m, y)}(b)\right)+\left(d_{Q(m, y)}(a) d_{Q(m, y)}(b)\right)\right]^{2} \\
= & \frac{m(y-1)(y-2)\left(y^{2}-1\right)^{2}}{2}+m(y-1)\left(y^{2}+y m-n-1\right)^{2} \\
& +\frac{m(y-1)(m+y-2)(m+y)^{2}}{2} .
\end{aligned}
$$

4. By making use the definition of $\mathrm{HGO}_{2}$ and values in Table 3 , we have

$$
\begin{aligned}
H G O_{2}(Q(m, y))= & \sum_{a b \in E(Q(m, y))}\left[\left(d_{Q(m, y)}(a)+d_{Q(m, y)}(b)\right)\left(d_{Q(m, y)}(a) d_{Q(m, y)}(b)\right)\right]^{2} \\
= & 2 m(y-1)^{7}(y-2)+m(y-1)[(m+2 y-3)(y-1)(m+y-2)]^{2} \\
& +2 m(y-1)(m+y-2)^{6} .
\end{aligned}
$$

The join of each cycle of length $m \geq 3$ and a new vertex in $C_{m}^{n}$. That is $\left(C_{m}+K_{1}\right)$. We term it a wheel chain. $W_{m}^{n}$ is the symbol for it. $G O_{1}, G O_{2}, H G O_{1}$ and $\mathrm{HGO}_{2}$ of $W_{m}^{n}$ are derived in the following theorem.

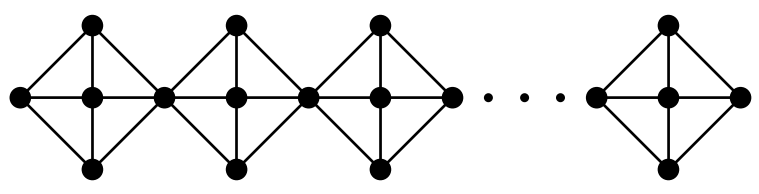

FiguRE 6. The graph $W_{4}^{n}$.

TABle 4. Partitioning at the edge of $W_{m}^{n}$.

\begin{tabular}{|c|c|}
\hline$d_{W_{m}^{n}}(a), d_{W_{m}^{n}}(b): a b \in E\left(W_{m}^{n}\right)$ & Edge count \\
\hline$(3,3)$ & $m n-4 n+4$ \\
\hline$(3,6)$ & $4(n-1)$ \\
\hline$(3, m)$ & $m n-2 n+2$ \\
\hline$(6, m)$ & $2(n-1)$ \\
\hline
\end{tabular}

Theorem 2.8. For wheel chain $W_{m}^{n}(m \geq 3, n \geq 2)$,

1. $G O_{1}\left(W_{m}^{n}\right)=4 m^{2} n+24 m n+54 n-6 m-54$

2. $G O_{2}\left(W_{m}^{n}\right)=3 m^{3} n+15 m^{2} n-6 m^{2}+108 m n+432 n-54 m-432$.

3. $H G O_{1}\left(W_{m}^{n}\right)=16 m^{3} n+354 n m+2070 n+90 m^{2} n-66 m^{2}-120 m-2070$.

4. $H_{G O_{2}}\left(W_{m}^{n}\right)=9 n m^{5}+108 n m^{4}+837 n m^{3}+2430 n m^{2}-72 m^{4}-864 m^{3}$

$-2592 m^{2}+2916 n m+93312 n-93312$. 
Proof. 1. By making use the definition of $G O_{1}$ and values in Table 4, we have

$$
\begin{aligned}
G O_{1}\left(W_{m}^{n}\right)= & \sum_{a b \in E\left(W_{m}^{n}\right)}\left[\left(d_{W_{m}^{n}}(a)+d_{W_{m}^{n}}(b)\right)+\left(d_{W_{m}^{n}}(a) d_{W_{m}^{n}}(b)\right)\right] \\
= & (m n-4 n+4)[6+9]+4(n-1)[9+18] \\
& +(m n-2 n+2)[3+m+3 m]+2(n-1)[6+m+6 m] \\
= & 4 m^{2} n+24 m n+54 n-6 m-54 .
\end{aligned}
$$

2. By the usage of the definition of $\mathrm{GO}_{2}$ and facts in Table 4, we have

$$
\begin{aligned}
G O_{2}\left(W_{m}^{n}\right)= & \sum_{a b \in E\left(W_{m}^{n}\right)}\left[\left(d_{W_{m}^{n}}(a)+d_{W_{m}^{n}}(b)\right)\left(d_{W_{m}^{n}}(a) d_{W_{m}^{n}}(b)\right)\right] \\
= & (m n-4 n+4)[6 \times 9]+4(n-1)[9 \times 18] \\
& +(m n-2 n+2)[(3+m) 3 m]+2(n-1)[(6+m) 6 m] \\
= & 3 m^{3} n+15 m^{2} n-6 m^{2}+108 m n+432 n-54 m-432 .
\end{aligned}
$$

3. By using the expression for $H G O_{1}$ and data in Table 4, we have

$$
\begin{aligned}
H G O_{1}\left(W_{m}^{n}\right)= & \sum_{a b \in E\left(W_{m}^{n}\right)}\left[\left(d_{W_{m}^{n}}(a)+d_{W_{m}^{n}}(b)\right)+\left(d_{W_{m}^{n}}(a) d_{W_{m}^{n}}(b)\right)\right]^{2} \\
= & (m n-4 n+4)[6+9]^{2}+4(n-1)[9+18]^{2} \\
& +(m n-2 n+2)[3+m+3 m]^{2}+2(n-1)[6+m+6 m]^{2} \\
= & 16 m^{3} n+354 n m+2070 n+90 m^{2} n-66 m^{2}-120 m-2070 .
\end{aligned}
$$

4. By utilizing the description of $\mathrm{HGO}_{2}$ and entries in Table 4, we have

$$
\begin{aligned}
H G O_{2}\left(W_{m}^{n}\right)= & \sum_{a b \in E\left(W_{m}^{n}\right)}\left[\left(d_{W_{m}^{n}}(a)+d_{W_{m}^{n}}(b)\right)\left(d_{W_{m}^{n}}(a) d_{W_{m}^{n}}(b)\right)\right] \\
= & (m n-4 n+4)[6 \times 9]^{2}+4(n-1)[9 \times 18]^{2} \\
& +(m n-2 n+2)[(3+m) 3 m]^{2}+2(n-1)[(6+m) 6 m]^{2} \\
= & 9 n m^{5}+108 n m^{4}+837 n m^{3}+2430 n m^{2}-72 m^{4}-864 m^{3}-2592 m^{2} \\
& +2916 n m+93312 n-93312 .
\end{aligned}
$$

\section{COMPARATIVE ANALYSIS}

The plotting of $G O_{1}, G O_{2}, H G O_{1}$ and $H G O_{2}$ for the cactus graphs are shown in Figures 7 and 8 . We have built the figures using Origin software taking $\mathrm{m}=4$. $G O_{1}\left(C_{m}^{n}\right), G O_{1}\left(C_{m}^{n}\right), G O_{1}\left(C O_{m}^{n}\right), G O_{1}\left(W_{m}^{n}\right), G O_{2}\left(C_{m}^{n}\right), G O_{2}\left(C O_{m}^{n}\right)$, $G O_{2}\left(W_{m}^{n}\right), H G O_{1}\left(C_{m}^{n}\right), H G O_{1}\left(C O_{m}^{n}\right), H G O_{1}\left(W_{m}^{n}\right), H G O_{2}\left(C_{m}^{n}\right), H G O_{2}\left(C O_{m}^{n}\right)$ 

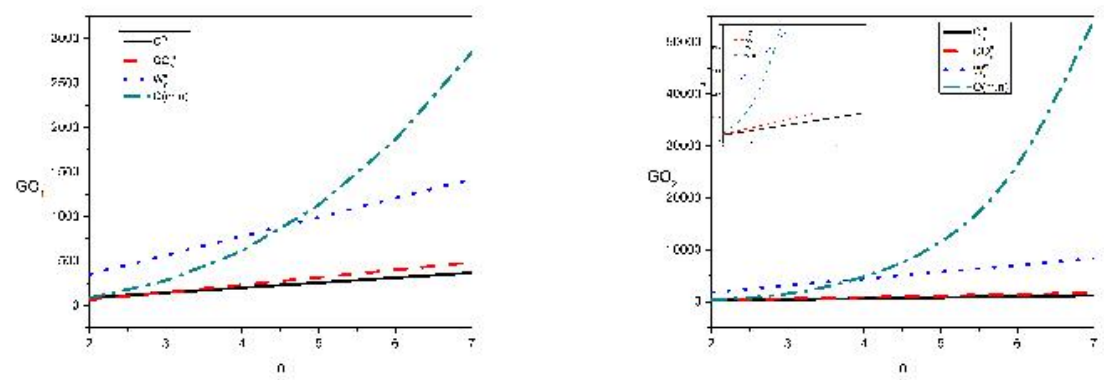

Figure 7. Plot of $G O_{1}$ (left) and $G O_{2}$ (right) for cactus chains.
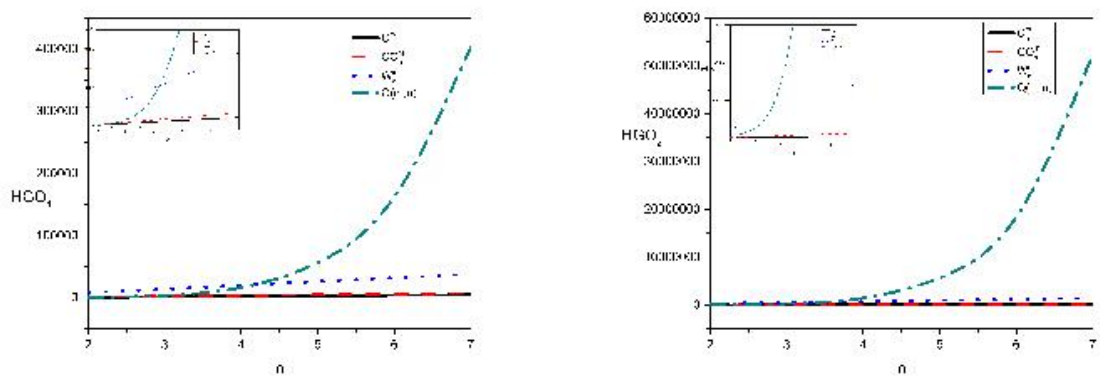

Figure 8. Plot of $\mathrm{HGO}_{1}$ (left) and $\mathrm{HGO}_{2}$ (right) for cactus chains.

and $\mathrm{HGO}_{2}\left(W_{m}^{n}\right)$ are linearly increasing and $\mathrm{GO}_{1}(Q(m, y)), G O_{2}(Q(m, y)), H G O_{1}$ $(Q(m, y))$ and $\mathrm{HGO}_{2}(Q(m, y))$ are exponentially increasing.

\section{CONCLUDING REMARKS}

In this paper, para cactus chain, ortho cactus chain and wheel cactus chain are discussed and explicit expressions of $G O_{1}, G O_{2}, H G O_{1}$ and $H G O_{2}$ are derived for them.

\section{REFERENCES}

[1] Alikhani, S., Jahari, S., Mehryar, M. and Hasni, R., Counting the number of dominating sets of cactus chains, Opt. Adv. Mat. Rapid Commun., 8(9-10) (2014), 955-960.

[2] Basavanagoud, B. and Policepatil, S., Chemical applicability of Gourava and hyper-Gourava indices, Nanosystems: Physics, Chemistry, Mathematics, 12(2) (2021), 142-150. 
[3] De, N., General Zagreb index of some cactus chains, Open Journal of Discrete Applied Mathematics, 2(1) (2019), 24-31.

[4] Gutman, I. and Trinajstić, N., Graph theory and molecular orbitals, Total $\pi$-electron energy of alternant hydrocarbons, Chem. Phys. Lett., 17 (4) (1972), 535-538.

[5] Harary, F., Graph Theory, Addison-Wesely, Reading Mass, 1969.

[6] Kulli, V. R., The Gourava indices and coindices of graphs, Annals of Pure and Applied Mathematics, 14(1) (2017), 33-38.

[7] Kulli, V. R., On hyper-Gourava indices and coindices, International Journal of Mathematical Archieve, 8(12) (2017), 116-120.

[8] Kulli, V. R., The Product connectivity Gourava index, Journal of Computer and Mathematical Science, 8(6) (2017), 235-242.

[9] Kulli, V. R., On the sum connectivity Gourava index, International Journal of Mathematical Archieve, 8(6) (2017), 211-217.

[10] Kulli, V. R., Computation of some Gourava indices of titania nanotubes, Intern. J. Fuzzy Mathematical Archieve, 12(2) (2017), 75-81.

[11] Kulli, V. R., Some Gourava indices and inverse sum indeg index of certain networks, International Research Journal of Pure Algebra, 7(7) (2017), 787-798.

[12] Mirajkar, K. G., and Pooja, B., On Gourava indices of some chemical graphs, International Journal of Applied Engineering Research, 14(3) (2019), pp. 743-749.

[13] Sadeghieh, A., Alikhani, S., Ghanbari N., and Khalaf, A. J. M., Hosoya polynomial of some cactus chains, Cogent Mathematics and statistics, 4(1), 1305638.

[14] Sadeghieh, A., Ghanbari, N., and Alikhani, S., Computation of Gutman index of some cactus chains, Electron. J. Graph Theory Appl., 6(1) (2018), 138-151. 